OPEN ACCESS

Edited by:

Jinbo Hu,

First Affiliated Hospital of Chongqing

Medical University, China

Reviewed by:

Alessandro Pocai,

Janssen Research and Development,

United States

Vahidreza Ostadmohammadi,

Kashan University of Medical

Sciences, Iran

*Correspondence:

Jirong Yue

yuejirong11@hotmail.com

Lunzhi Dai

lunzhi.dai@scu.edu.cn

Specialty section:

This article was submitted to

Endocrinology of Aging,

a section of the journal

Frontiers in Endocrinology

Received: 15 June 2021

Accepted: 24 August 2021

Published: 13 September 2021

Citation:

Dai M, Lin T, Yue J and Dai L (2021)

Signatures and Clinical Significance of

Amino Acid Flux in Sarcopenia: A

Systematic Review and Meta-Analy.

Front. Endocrinol. 12:725518.

doi: 10.3389/fendo.2021.725518

\section{Signatures and Clinical Significance of Amino Acid Flux in Sarcopenia: A Systematic Review and Meta-Analysis}

\author{
Miao Dai ${ }^{1}$, Taiping Lin ${ }^{1}$, Jirong Yue ${ }^{1 *}$ and Lunzhi Dai ${ }^{2 *}$ \\ ${ }^{1}$ Department of Geriatrics and National Clinical Research Center for Geriatrics, West China Hospital of Sichuan University, \\ Chengdu, China, 2 Department of State Key Laboratory of Biotherapy, West China Hospital of Sichuan University, and \\ Collaborative Innovation Center of Biotherapy, Chengdu, China
}

Background: Dysregulation of amino acids is closely linked to the initiation and progression of sarcopenia. We summarized recent advancements in the studies of amino acid profiles in sarcopenia and systematically presented the clinical significance of amino acid flux in sarcopenia.

Methods: We systematically searched in MEDLINE, EMBASE, and Cochrane library from inception to June 1, 2021 to capture all studies examining metabolomics of sarcopenia. We used the following keywords: sarcopenia, metabonomics, metabolomics, amino acid profile, and mass spectrometry. Original articles comparing amino acid patterns between persons with and without sarcopenia were included. Two independent investigators independently completed title and abstract screening, data extraction, and quality evaluation. We used a random effects model to examine the association between amino acids levels and sarcopenia. Sensitivity analyses restricted the analyses to studies in which muscle mass was measured by bioelectrical impedance analysis. Study quality was evaluated according to the Agency for Healthcare Research and Quality (AHRQ) checklist.

Results: The systematic research yielded six eligible articles, comprising 1,120 participants. Five studies used muscle mass in combination with physical performance and/or muscle strength as the criteria to diagnose sarcopenia, while one study used muscle mass as a diagnostic criterion alone. We found that the concentrations of branched-chain amino acids leucine (standardized mean difference [SMD] -1.249; 95\% confidence interval [Cl]: $\left.-2.275,-0.223, P=0.02, I^{2}=97.7 \%\right)$, isoleucine (SMD -1.077; 95\% Cl: $-2.106,-0.049, P=0.04, I^{2}=97.8 \%$ ), and aromatic amino acid tryptophan (SMD $\left.-0.923 ; 95 \% \mathrm{Cl}:-1.580,-0.265, P=0.01, \mathrm{I}^{2}=89.9 \%\right)$ were significantly reduced in individuals with sarcopenia. Study results were robust in sensitivity analysis.

Conclusions: The homeostasis of amino acids is critical to maintaining muscle health. The profiles of amino acids might be useful biomarkers for the characterization of sarcopenia. Future studies are warranted to study the clinical significance of amino acids in the diagnosis and treatment of sarcopenia.

Keywords: sarcopenia, metabolomics, amino acid, biomarkers, meta-analysis 


\section{INTRODUCTION}

Sarcopenia, defined as the age-related progressive loss of skeletal muscle strength, mass, and/or function (1), has been established as a strong risk factor for falls $(2,3)$, disability $(4)$, low health-related quality of life, and premature death among older adults $(5,6)$. Skeletal muscle mass declines at an annual rate of 1-2\% among adults over 50 years, leading to decreased muscle strength and function (7). The direct and indirect medical costs associated with sarcopenia are expected to rise rapidly in the era of population aging, resulting in a substantial financial burden for older adults, their caregivers, and the healthcare system. However, the molecular mechanism and etiology of sarcopenia remain unclear. Understanding the pathogenesis of sarcopenia is urgently needed to improve its prevention, diagnosis, treatment, and prognosis.

Metabolites represent the downstream expression of the genome, transcriptome, and proteome and are closely related to cellular phenotypes. Mass spectrometry-based metabolomics emerges as a robust approach to systematically analyze metabolic profiles in tissues, biological fluids, and cells (8), revealing the significance of metabolic flux in various diseases, including cancer (9), aging, and longevity $(10,11)$. Of the metabolites, the investigation of amino acids is essential. Understanding the amino acid profile changes may contribute to a better understanding of pathophysiological mechanisms.

Identifying amino acid profiles associated with sarcopenia may help identify at-risk populations, optimize prevention strategies, and develop new treatments. Previous studies have shown that changes in the plasma amino acid profile were associated with low muscle mass among older adults (12). The metabolism of amino acids, such as aspartic acid and glutamic acid, might play an essential role in regulating muscle mass and strength (13). There is evidence that lower blood levels of essential amino acids (EAA), branched-chain amino acids (BCAAs), especially leucine, were associated with lower skeletal muscle index (SMI), strength, and longer time to complete the chair stand (14). However, a study showed that the levels of isoleucine, leucine, tryptophan, serotonin, and methionine in the participants with low muscle quality were significantly higher than that in the participants with high muscle quality, which may be attributed to impaired metabolism of amino acids, resulting in reduced uptake of skeletal muscle, and thus increased circulating plasma amino acid levels (15). Inconsistencies in amino acid profiles in patients with sarcopenia will lead to variations in clinical practice and research.

Therefore, we conducted a systematic review and metaanalysis to evaluate and synthesize the evidence regarding the association between amino acid profile and sarcopenia in the present study.

\section{METHODS}

\section{Data Sources and Search Strategy}

A comprehensive search was conducted on MEDLINE, EMBASE, and Cochrane library via Ovid SP for all publications related to amino acid metabolomics biomarkers of sarcopenia reported from inception to June 1, 2021. The terms sarcopenia, metabonomics, metabolomics, amino acid profile, and mass spectrometry were searched alone or in combination. Take the MEDLINE search policy as an example. The search strategies are presented in Table 1. A manual search for additional potentially applicable studies was carried out by using references of included studies. No language limitations were applied. The study followed the Preferred Reporting Items for Systematic Reviews and MetaAnalyses (PRISMA) flow diagram and checklist (16).

\section{Inclusion and Exclusion Criteria}

We used the following inclusion criteria for each included manuscript: (1) Studies must have been conducted on adults (age $\geq 50$ years). (2) A definition of sarcopenia was described in the methods section. The diagnostic criteria for sarcopenia included muscle mass, muscle strength, and/or body function. (3) It was an original article, including observational studies (cross-sectional studies, cohort studies, and case-control studies) and experimental studies (randomized controlled trials).

Articles that met the following criteria were excluded: (1) Studies performed in animals, case reports, reviews, conference abstracts, letters to the Editor, and books. (2) Therapeutic trials and articles used methods other than metabolomics.

\section{Data Extraction and Quality Assessment}

Two investigators (MD and TL) independently filtered these articles' titles and abstracts and retrieved the full text based on our inclusion and exclusion criteria to ensure accuracy. Disagreement in screening the articles was resolved by discussion between the two investigators, and if necessary, involving a third investigator (JY).

Two investigators extracted and summarized study characteristics in a standard form independently. Any disagreement was resolved by consensus. The following information was extracted from the eligible studies: name of the first author, year of publication, study population, study design, sample size, the mean age of individuals,

TABLE 1 | Search strategy.

\section{MEDLINE(R)}

Database: Ovid MEDLINE(R) Epub Ahead of Print, In-Process \& Other NonIndexed Citations, Ovid MEDLINE(R) Daily, and Ovid MEDLINE(R)

1 exp Sarcopenia

2 (sarcopeni* or myopeni* or dynapeni*).ti,ab.

3 [(muscle or muscular) adj2 (atroph* or wasting* or weak or loss*)].ti,ab.

4 exp Metabolomics

5 exp Metabonomics

6 (metabolo* OR metabolomic* OR metabonomic* OR liquid chromatogra* OR gas chromatogra* OR ultra-performance liquid chromatograph ${ }^{\star} \mathrm{OR}$ high performance liquid chromatograph* $\mathrm{OR}$ high-performance liquid chromatograph*).ti,ab.

7 (metabolit OR lipidomic OR UPLC OR proton nuclear magnetic resonance OR proton NMR OR nuclear magnetic resonance spectrometry OR H NMR OR mass spectrometry OR nuclear magnetic spectroscopy OR metabolic profiling OR amino acid profile OR amino acid metabolomics OR amino acid metabonomics OR amino acid metabolism).ti,ab.

8 ( 1 or 2 or 3$)$ and ( 4 or 5 or 6 or 7 )

Asterisks $\left(^{*}\right)$ indicate truncation symbol. 
percentage of females, diagnostic methods and specific criteria for sarcopenia, assessment method of muscle strength and muscle mass and physical performance, metabolomics techniques and metabolite targets, biological specimen (plasma or serum), and major metabolite outcomes. The quality of cross-sectional studies was assessed according to the Agency for Healthcare Research and Quality (AHRQ) checklist. There are 11 items in the AHRQ checklist; each item has three responses: "Yes," "No," and "Unclear". A score of 0 was assigned to an answer of "No" or "Unclear", while a score of 1 was given to an answer of "Yes". Study quality was assessed based on the total score (range: 0-11) and classified into three categories: low (score: 0-3), moderate (score: 4-7), and high (score: 8-11).

\section{Data Synthesis and Analysis}

We extracted the mean and standard deviation of concentrations of amino acids from each study. The median (range) was converted into average and standard deviation following a method published previously (17). We used the random effects models, which considered between- and within-study variability, to pool the association estimates between concentrations of amino acids and sarcopenia. We used the Cochran $\mathrm{Q}$ test ( $P<0.1$ indicates statistically significant heterogeneity) and the $\mathrm{I}^{2}$ statistic $\left(\mathrm{I}^{2}>50 \%\right.$ indicates statistically significant heterogeneity) to evaluate study heterogeneity. Forest plots were used to visualize the results. Standardized mean difference
(SMD) with 95\% confidence interval (CI) was selected for the continuous outcomes. As a sensitivity analysis, we restricted the analyses to studies in which muscle mass was measured by bioelectrical impedance analysis (BIA). We only analyzed amino acid metabolites that were used in at least two studies. We did not assess publication bias because fewer than 10 eligible studies were included. All statistical analyses were performed using Stata, version 12. A two-tailed P value of $<0.05$ was considered statistically significant.

\section{RESULTS}

\section{Literature Information}

In the initial search, 991 articles were retrieved from MEDLINE, EMBASE, and Cochrane. Six hundred and eighty-two studies were identified after eliminating duplicates. We further excluded 659 studies after following the review of titles and abstracts because they were not relevant to the subject, or were conducted in animals, or were non-original studies. Twenty-three articles were selected for careful full-text screening. Full-text articles were further excluded if they were not relevant to the topic or did not meet inclusion criteria. In total, six full-text articles were included in this systematic review and meta-analysis (Figure 1).

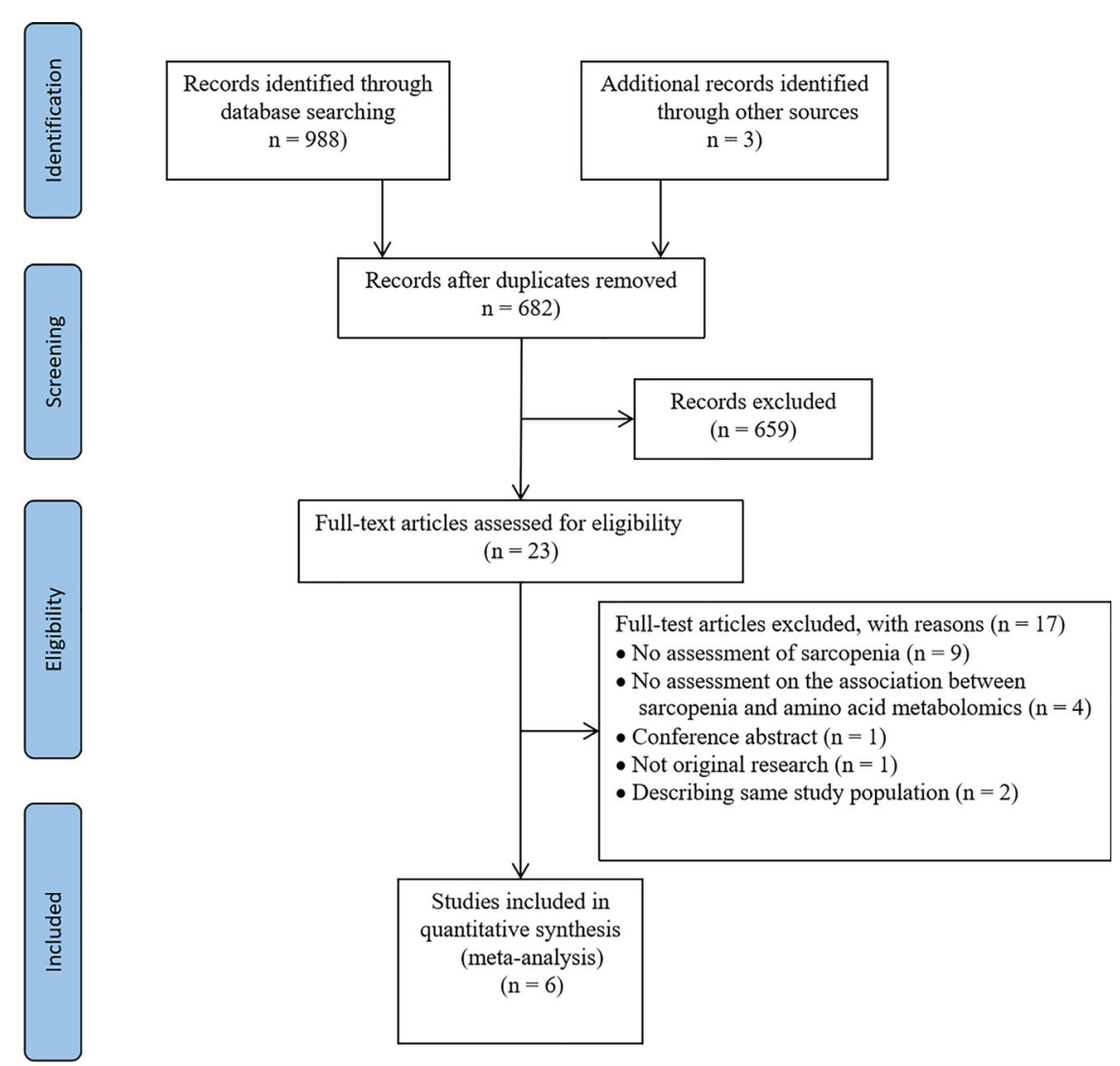

FIGURE 1 | Flow of the search strategy and included studies. 


\section{Characteristics of Included Studies}

Of the 991 screened papers, six papers were eligible (Figure 1). A total of 1,120 participants were included. All studies compared the amino acids levels in those with sarcopenia (case subjects) versus those without (control subjects). Sample size ranged from 28 to 90 in the case groups $(n=358)$ and 30 to 327 in the control groups $(n=762)$. Five studies included participants of both sexes; one study included women only (18). Five studies used LMM in combination with low physical performance (LPP) and/or low muscle strength (LMS) as the criteria for diagnosing of sarcopenia (18-22), while one study used only LMM as the diagnostic criterion (23). Muscle mass was measured by BIA in three studies $(18,19,21)$, dual-energy X-ray absorptiometry (DXA) in two studies $(20,22)$, and computed tomography in one study (23). Muscle strength was measured through handgrip dynamometry in three studies $(18,19,21)$, and lower limb strength in one study (20). Physical performance was measured via gait speed in five studies (18-22). Muscle mass, muscle strength, and physical performance were assessed according to cut-off thresholds recommended by the Asian Working group for Sarcopenia in three studies (18-20), European Working Group for Sarcopenia in Older people (EWGSOP) in one study (21), and the Foundation for the National Institutes of Health (FNIH) in one study (22), while only one study evaluated sarcopenia by measuring psoas muscle area at the caudal end of the third lumbar vertebra by computed tomography (CT) without fulfilling specific guidelines (23). Metabolite analysis was performed using blood samples; five used plasma and one used serum. All studies used targeted metabolomics for analyzing metabolite features. One study used high-performance liquid chromatography-tandem mass spectrometry (HPLC-MS/MS) (19), one study employed nuclear magnetic resonance spectroscopy (NMR) (21), and one study used ultra-performance liquid chromatography-mass spectrometry (UPLC-MS) (22), while the techniques used in other studies were unclear. Five studies' main results were presented as mean and standard deviation $(18,20-23)$, and the remaining one study reported the correlation between sarcopenia and metabolites with median (range) (19). The characteristics of each eligible study are summarized in Table 2.

\section{Quality Assessment}

We assessed the quality of all six eligible studies according to the AHRG checklist. All studies were of moderate quality (score: 36); five studies had a score of 5 and one scored 6 (Table 3).

\section{Meta-Analysis of Remarkable Metabolites}

Eighteen metabolites that were included in at least two studies were further analyzed by pooled meta-analysis using a randomeffects model (Figure 2). The concentrations of BCAAs leucine (SMD -1.249, 95\% CI: -2.275, -0.223), isoleucine (SMD -1.077, 95\% CI: $-2.106,-0.049$ ), and aromatic amino acid tryptophan (SMD -0.923, 95\% CI: $-1.580,-0.265$ ) were significantly lower in participants with sarcopenia than in those without, but the heterogeneity was high for each amino acid (leucine: $\mathrm{I}^{2}=$ 97.7\%; isoleucine: $\mathrm{I}^{2}=97.8 \%$; tryptophan: $\mathrm{I}^{2}=89.9 \%$ ).

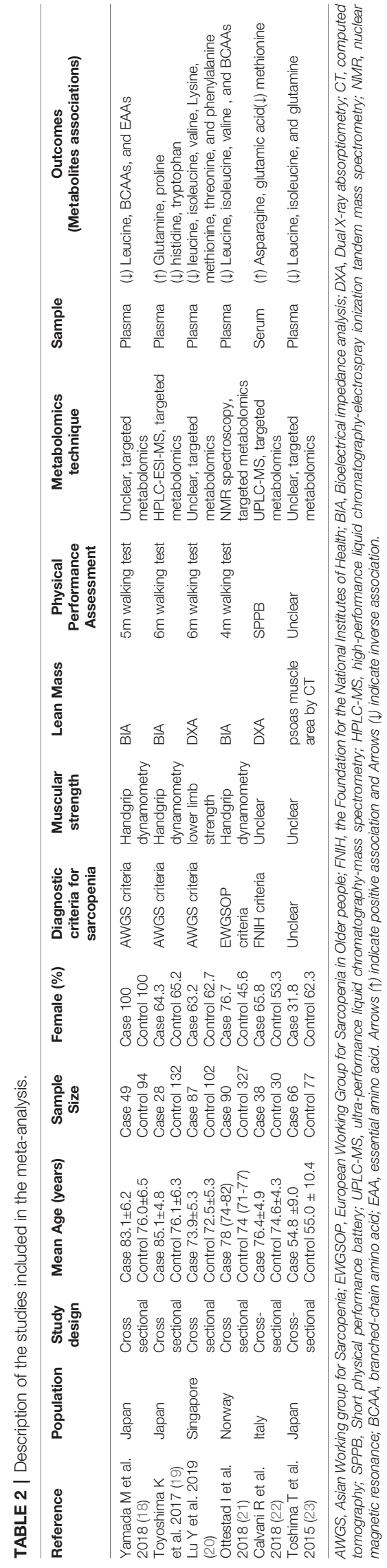


TABLE 3 | AHRQ checklist for assessing the quality of cross-sectional studies.

\begin{tabular}{|c|c|c|c|c|c|c|c|c|c|c|c|c|c|}
\hline Study & Q1 & Q2 & Q3 & Q4 & Q5 & Q6 & Q7 & Q8 & Q9 & Q10 & Q11 & Score & Quality \\
\hline (18) & + & + & + & $U$ & $U$ & + & - & + & - & + & - & 5 & Moderate \\
\hline (19) & + & - & + & $U$ & $U$ & + & - & + & - & + & - & 5 & Moderate \\
\hline (20) & + & - & + & U & $U$ & + & - & + & - & + & - & 5 & Moderate \\
\hline (21) & + & + & + & $U$ & $U$ & + & + & + & - & + & - & 6 & Moderate \\
\hline (22) & + & + & + & U & $U$ & + & - & - & - & + & - & 5 & Moderate \\
\hline (23) & + & - & + & $U$ & $U$ & + & + & - & - & + & - & 5 & Moderate \\
\hline
\end{tabular}

AHRQ, Agency for Healthcare Research and Quality; +, yes; -, no; U, unclear.

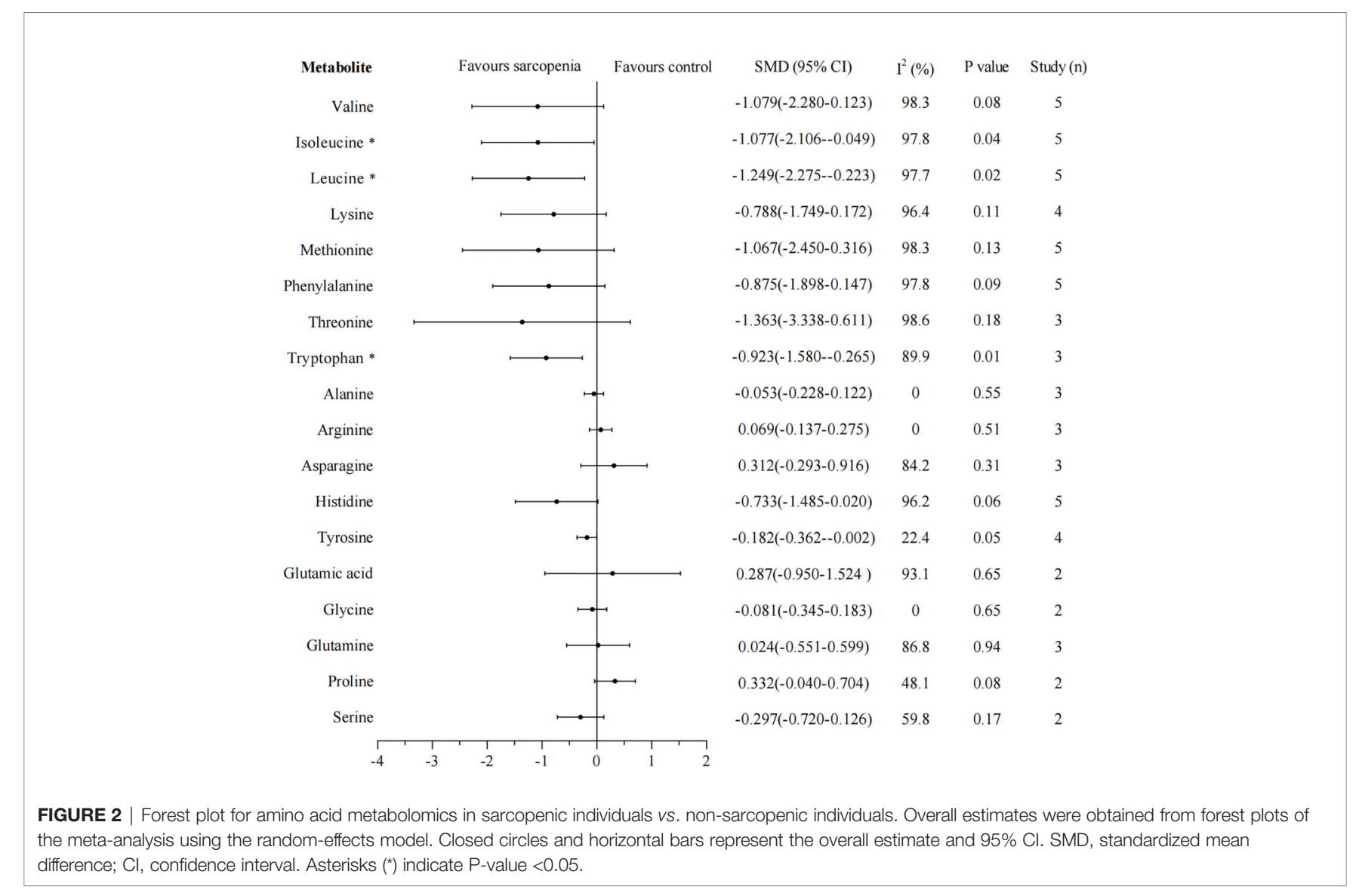

\section{Sensitivity Analysis}

We rerun the random effects model excluding studies that did not measure muscle mass through BIA. Thirteen metabolites that were included in at least two studies were analyzed separately. The results of leucine, isoleucine, tryptophan, lysine, methionine, arginine, asparagine, tyrosine, and glutamine were unchanged, and the heterogeneity greatly reduced in studies except for arginine and tyrosine, $\mathrm{I}^{2}$ were $0 \%, 0 \%, 55.7 \%, 0 \%, 0 \%, 17.6 \%, 0 \%, 47.9 \%, 44.7 \%$, respectively (Figure 3). However, the results of valine, phenylalanine, threonine, and histidine changed, their concentrations were significantly lower in sarcopenia than in non-sarcopenia (valine: SMD -0.294, 95\% CI: -0.469, -0.118; phenylalanine: SMD -0.215, 95\% CI: $-0.390,-0.040$; threonine: SMD $-0.278,95 \%$ CI: $-0.543,-0.014$; histidine: SMD $-0.285,95 \%$ CI: $-0.460,-0.110$ ), these four metabolites showed no significant evidence of heterogeneity (All $\mathrm{I}^{2}=0 \%$ ).

\section{DISCUSSION}

We performed a meta-analysis of literature related to sarcopenia's metabolic profiling and highlighted the clinical significance of amino acids flux in sarcopenia. Our results from cross-sectional studies support inverse associations of the BCAAs leucine, isoleucine, and aromatic amino acid tryptophan with sarcopenia, which may provide insights into the development of sarcopenia and contribute to the prevention and treatment of sarcopenia.

\section{Compared With Other Studies}

The significant roles of amino acids in maintaining skeletal muscle function and muscle protein synthesis have been disclosed in previous studies (24-26). In functionally limited older adults, the BCAA levels were significantly positively associated with thigh 


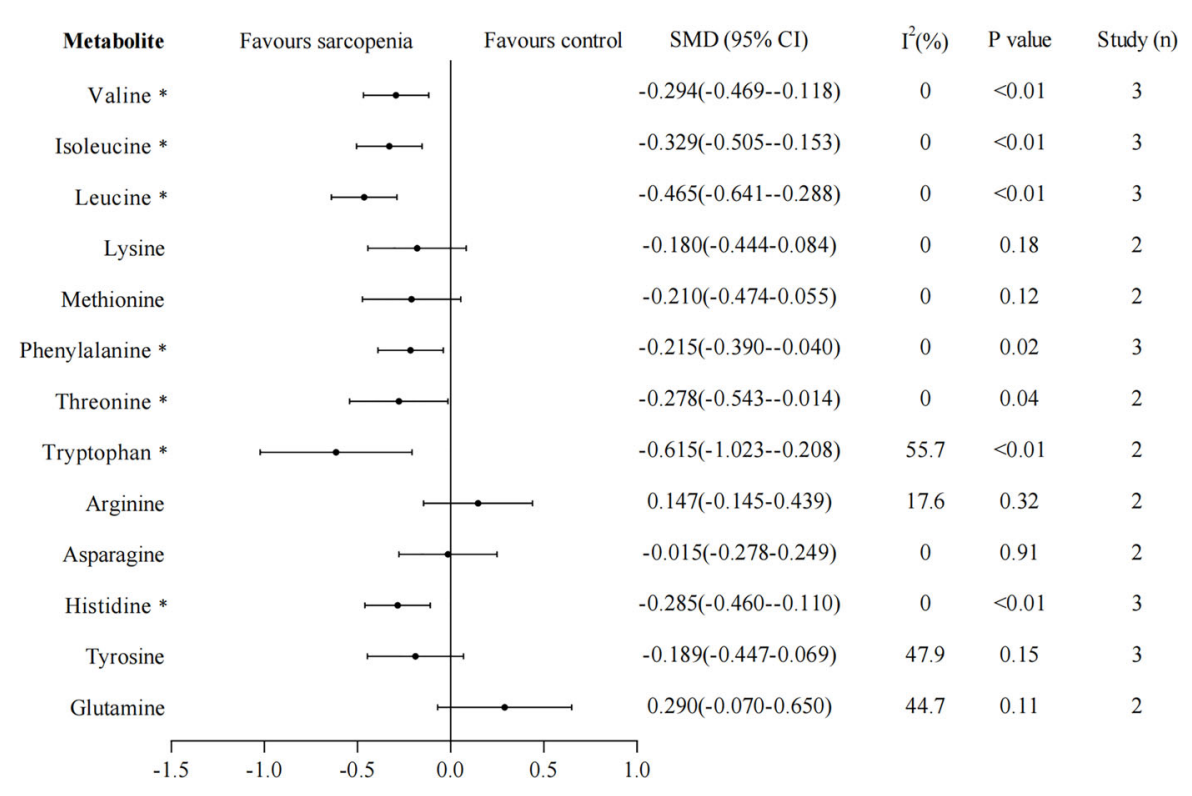

FIGURE 3 | Sensitivity analyses for amino acid metabolomics in sarcopenic individuals vs. non-sarcopenic individuals (restricting to studies using bioelectrical impedance analysis to measure muscle mass). Overall estimate obtained from forest plots of the random-effects meta-analysis using the SMD. Closed circles and horizontal bars represent the overall estimate and 95\% Cl. SMD, standardized mean difference. Cl, confidence interval. Asterisks $\left({ }^{\star}\right)$ indicate P-value $<0.05$.

muscle cross-sectional area (CSA) and fat-free mass index (FFMI) (12). In our study, we found that leucine and isoleucine levels but not valine in participants with sarcopenia were significantly lower than those with non-sarcopenia, but high heterogeneity occurred across the integrated literature. Leucine and isoleucine are important essential amino acids that can only be obtained from food and are important nutritional factors for humans and animals (27). Studies have shown that the target of rapamycin complex 1 (mTORC1) kinase is a sensor of amino acids. Sestrin 1-3 interact with GATOR2 to negatively regulate the amino acid-sensitive pathway upstream of mTORC1 $(28,29)$. Whereas leucine stimulation leads to the dissociation of Sestrin 2 from GATOR2 and allows the activation of mTORC1 pathway (30), which further activates of eukaryotic initiation factor (eIF) 4E binding protein-1 and ribosomal protein S6 kinase (S6K1), and promotes the muscle protein synthesis (Figure 4) (31-34). Additionally, Liu S et al. (35) revealed that isoleucine increased the protein level of important myoblast genes and promoted myoblast proliferation and myoblast differentiation, suggesting that it has a potential function in myogenesis. A study indicated that the reduced muscle protein synthesis in the old adults could be reversed by the intake of additional leucine and the leucine-rich mixture of EAAs (36). Furthermore, with physical exercise, leucine-rich EAA supplementation effectively improved skeletal muscle mass volume, muscle strength, and walking speed in sarcopenic women (37). These findings suggest that decreased leucine and isoleucine concentrations may contribute to sarcopenia development and maintaining optimizing concentrations of EAAs in plasma, especially leucine and isoleucine, may be beneficial to muscle protein synthesis and very important for maintaining physical function in the sarcopenic elderly.
The aromatic amino acids include tryptophan, phenylalanine, and tyrosine, of which tryptophan and phenylalanine are EAAs. A previous study has shown that tryptophan can significantly affect muscle mass by its metabolite serotonin, and animals deficient in tryptophan showed low growth hormone $(\mathrm{GH})$ levels and significant muscle atrophy (38). Dukes A et al. (39) confirmed that tryptophan stimulates skeletal muscle IGF1/p70s6k/mTOR signaling in vivo and can induce the expression of myogenic factors (myogenin, myoD, and myosin heavy chain) in $\mathrm{C} 2 \mathrm{C} 12$ myoblasts in vitro, which play a prominent role in the regulation of myofiber size and muscle mass. Serum tryptophan decreases with age in older men (40). In our study, plasma tryptophan concentration was significantly lower in sarcopenic people than in non-sarcopenic people. Also, there is evidence that dietary supplementation with tryptophan could stimulate muscle protein synthesis in swine (38). It may be possible that the decrease of tryptophan leads to increased skeletal muscle cell atrophy and affects muscle mass, thus promoting sarcopenia. Therefore, we infer that the tryptophan metabolic pathway may be a promising target for preventing or treating skeletal muscle atrophy. Nevertheless, the findings were presented in cross-sectional studies. Thus, a longitudinal study is needed to clarify the role of tryptophan in the development of sarcopenia.

\section{Heterogeneity and Sensitivity Analysis}

The reasons for the high heterogeneity may be that: (1) Methods of measuring muscle mass were different. A meta-analysis showed that BIA yielded higher sarcopenia prevalence estimates than DXA (41). Psoas is a minor muscle, it does not represent the whole muscle, and the cut-off point for low muscle mass is not well defined for the measurement of psoas muscle 


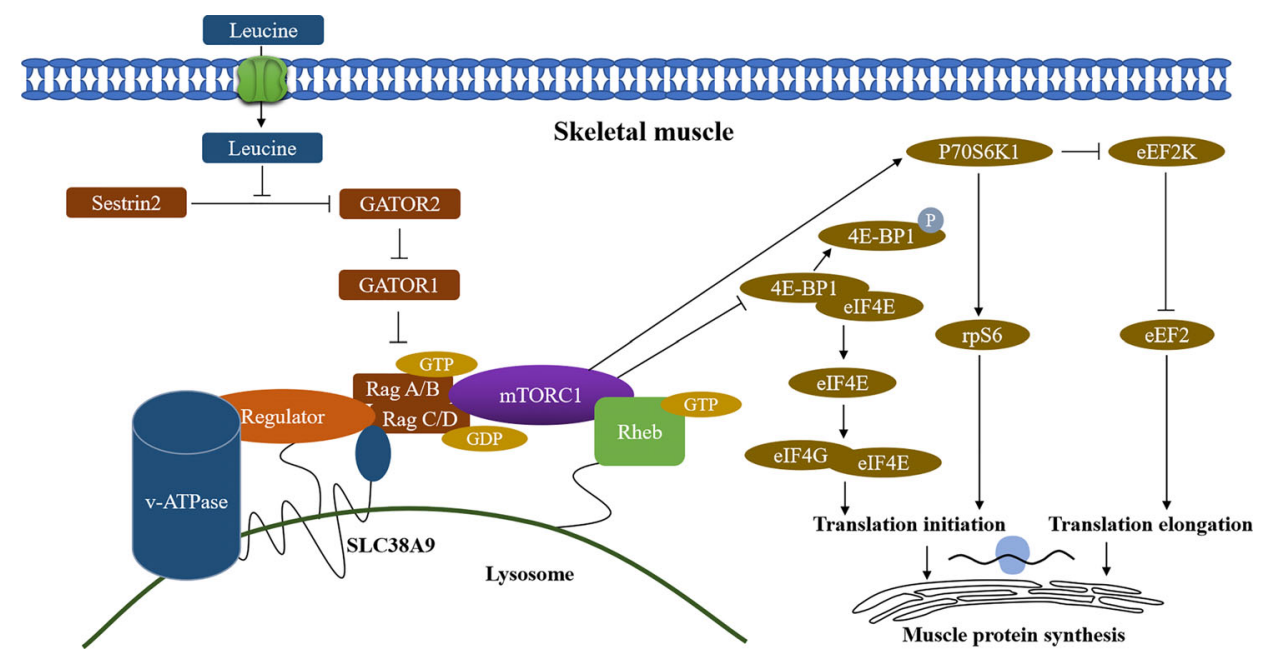

FIGURE 4 | Regulation of skeletal muscle protein synthesis by leucine. Sestrin2, stress response protein 2; GATOR1/2, GAP activity toward Rags 1/2; mTORC1, mammalian/mechanistic target of rapamycin complex 1; v-ATPase, vacuolar $\mathrm{H}+$-adenosine triphosphatase; Rag A/B C/D, RAS-related GTP-binding protein A/B C/D; SLC38A9, Solute Carrier Family 38 Member 9; 4E-BP1, 4E-binding protein 1; elF4E, eukaryotic initiation factor 4E; elF4G, eukaryotic initiation factor 4G; p70S6K1, p70 ribosomal protein S6 kinase 1; eEF2K, eukaryotic elongation factor 2 kinase; rpS6, ribosomal protein S6; eEF2, eukaryotic elongation factor 2; Arrows ( $\uparrow$ ) indicate stimulation and blocked lines $(-1)$ indicate inhibition.

area by CT (42). (2) The included studies were carried out in different places (such as communities, rural areas, hospitals, or nursing homes) and different countries, so ethnic and regional factors might also influence the results. (3) Different metabonomics profiling techniques were applied, and the metabolomics results may have testing errors. Compared with other techniques, NMR analysis requires a larger sample size, poor isolation, and background noise that may obscure potential biomarkers (43).

For the high heterogeneity, we conducted sensitivity analysis by excluding studies that did not use BIA to measure muscle mass. It showed that the results of leucine, isoleucine, tryptophan, lysine, methionine, arginine, asparagine, and glutamine were consistent with those of the preliminary analysis. However, the concentrations of valine, phenylalanine, threonine, and histidine in the case group became significantly lower than those in the control group. Sensitivity analysis also revealed that the heterogeneity greatly was reduced in studies, suggesting that these deleted studies may lead to high heterogeneity and instability of results. Whether valine, phenylalanine, threonine, histidine, and tyrosine are potential biomarkers for sarcopenia requires further studies. Due to the limited number of included articles, other causes of heterogeneity could not be analyzed.

\section{Strengths and Limitations}

To our knowledge, this was the first systematic review and metaanalysis to explore the association between amino acids and sarcopenia. Two investigators conducted the literature search, article screening, and data extraction independently to minimize bias. However, this study is not without limitations. First, our study's most significant limitation was that the metabolomics results showed that the high heterogeneity indexes accounted for
$2 / 3$. Second, our results are subjective to selection bias. The participants were mostly volunteers, not randomly selected, and there were more women than men in these studies. Third, although our search strategy is not limited to English, studies that were not indexed in EMBASE, MEDLINE, or Cochrane library might be excluded. Finally, all of these included studies were cross-sectional, and we were unable to identify the causal relationship between amino acid metabolites and sarcopenia.

\section{Conclusion}

In conclusion, reduced plasma concentrations of leucine, isoleucine, and tryptophan may signify sarcopenia. Determination of the optimal levels of plasma leucine, isoleucine, and tryptophan helps prevent and treat sarcopenia and maintain muscle mass and function. These findings need to be confirmed by further studies, including randomized trials and cohort studies.

\section{DATA AVAILABILITY STATEMENT}

The original contributions presented in the study are included in the article/supplementary material. Further inquiries can be directed to the corresponding authors.

\section{AUTHOR CONTRIBUTIONS}

MD designed the study protocol, extracted the data, designed and performed the analyses, interpreted the results, wrote the first drafts and revisions of manuscripts. TL contributed to the search strategy, study selection and extracted the data. JY revised drafts of the report, solved all disagreements and supervised the study. LD contributed in the interpretation of the findings and 
revised drafts of the report. All authors contributed to the article and approved the submitted version.

\section{FUNDING}

This study was supported by grants from National Key R\&D Program of China (No.2020YFC2005600 and 2020YFC2005602),

\section{REFERENCES}

1. Chen LK, Woo J, Assantachai P, Auyeung TW, Chou MY, Iijima K, et al. Asian Working Group for Sarcopenia: 2019 Consensus Update on Sarcopenia Diagnosis and Treatment. J Am Med Dir Assoc (2020) 21(3):300-7.e2. doi: 10.1016/j.jamda.2019.12.012

2. Landi F, Liperoti R, Russo A, Giovannini S, Tosato M, Capoluongo E, et al. Sarcopenia as a Risk Factor for Falls in Elderly Individuals: Results From the ilSIRENTE Study. Clin Nutr (2012) 31(5):652-8. doi: 10.1016/j.clnu. 2012.02.007

3. Bischoff-Ferrari HA, Orav JE, Kanis JA, Rizzoli R, Schlögl M, Staehelin HB, et al. Comparative Performance of Current Definitions of Sarcopenia Against the Prospective Incidence of Falls Among Community-Dwelling Seniors Age 65 and Older. Osteoporos Int (2015) 26(12):2793-802. doi: 10.1007/s00198015-3194-y

4. Clark BC, Manini TM. Functional Consequences of Sarcopenia and Dynapenia in the Elderly. Curr Opin Clin Nutr Metab Care (2010) 13 (3):271-6. doi: 10.1097/MCO.0b013e328337819e

5. Landi F, Cruz-Jentoft AJ, Liperoti R, Russo A, Giovannini S, Tosato M, et al. Sarcopenia and Mortality Risk in Frail Older Persons Aged 80 Years and Older: Results From ilSIRENTE Study. Age Ageing (2013) 42(2):203-9. doi: 10.1093/ageing/afs 194

6. Locquet M, Beaudart C, Hajaoui M, Petermans J, Reginster JY, Bruyère O. Three-Year Adverse Health Consequences of Sarcopenia in CommunityDwelling Older Adults According to 5 Diagnosis Definitions. J Am Med Dir Assoc (2019) 20(1):43-6. doi: 10.1016/j.jamda.2018.06.004

7. Hughes VA, Frontera WR, Roubenoff R, Evans WJ, Singh MA. Longitudinal Changes in Body Composition in Older Men and Women: Role of Body Weight Change and Physical Activity. Am J Clin Nutr (2002) 76(2):473-81. doi: 10.1093/ ajcn/76.2.473

8. Ceglarek U, Leichtle A, Brügel M, Kortz L, Brauer R, Bresler K, et al. Challenges and Developments in Tandem Mass Spectrometry Based Clinical Metabolomics. Mol Cell Endocrinol (2009) 301(1-2):266-71. doi: 10.1016/j.mce.2008.10.013

9. Gu Y, Chen T, Fu S, Sun X, Wang L, Wang J, et al. Perioperative Dynamics and Significance of Amino Acid Profiles in Patients With Cancer. J Transl Med (2015) 13:35. doi: 10.1186/s12967-015-0408-1

10. Collino S, Montoliu I, Martin FP, Scherer M, Mari D, Salvioli S, et al. Metabolic Signatures of Extreme Longevity in Northern Italian Centenarians Reveal a Complex Remodeling of Lipids, Amino Acids, and Gut Microbiota Metabolism. PloS One (2013) 8(3):e56564. doi: 10.1371/ journal.pone. 0056564

11. Montoliu I, Scherer M, Beguelin F, DaSilva L, Mari D, Salvioli S, et al. Serum Profiling of Healthy Aging Identifies Phospho- and Sphingolipid Species as Markers of Human Longevity. Aging (Albany NY) (2014) 6(1):9-25. doi: 10.18632/aging. 100630

12. Lustgarten MS, Price LL, Chale A, Phillips EM, Fielding RA. Branched Chain Amino Acids are Associated With Muscle Mass in Functionally Limited Older Adults. J Gerontol A Biol Sci Med Sci (2014) 69(6):717-24. doi: 10.1093/ gerona/glt152

13. Zhao Q, Shen H, Su KJ, Tian Q, Zhao LJ, Qiu C, et al. A Joint Analysis of Metabolomic Profiles Associated With Muscle Mass and Strength in Caucasian Women. Aging (Albany NY) (2018) 10(10):2624-35. doi: 10.18632/aging.101574

14. Ter Borg S, Luiking YC, van Helvoort A, Boirie Y, Schols JMGA, de Groot CPGM. Low Levels of Branched Chain Amino Acids, Eicosapentaenoic Acid and 1.3.5 project for disciplines of excellence, West China Hospital, Sichuan University (NO. ZY2017201).

\section{ACKNOWLEDGMENTS}

\section{We thank English language editor CKW for revising} the manuscript.

and Micronutrients Are Associated With Low Muscle Mass, Strength and Function in Community-Dwelling Older Adults. J Nutr Health Aging (2019) 23(1):27-34. doi: 10.1007/s12603-018-1108-3

15. Moaddel R, Fabbri E, Khadeer MA, Carlson OD, Gonzalez-Freire M, Zhang P, et al. Plasma Biomarkers of Poor Muscle Quality in Older Men and Women From the Baltimore Longitudinal Study of Aging. J Gerontol A Biol Sci Med Sci (2016) 71(10):1266-72. doi: 10.1093/gerona/glw046

16. Moher D, Liberati A, Tetzlaff J, Altman DGPRISMA Group. Preferred Reporting Items for Systematic Reviews and Meta-Analyses: The PRISMA Statement. BMJ (2009) 339:b2535-5. doi: 10.1136/bmj.b2535

17. Hozo SP, Djulbegovic B, Hozo I. Estimating the Mean and Variance From the Median, Range, and the Size of a Sample. BMC Med Res Methodol (2005) 5:13. doi: 10.1186/1471-2288-5-13

18. Yamada M, Kimura Y, Ishiyama D, Nishio N, Tanaka T, Ohji S, et al. Plasma Amino Acid Concentrations Are Associated With Muscle Function in Older Japanese Women. J Nutr Health Aging (2018) 22(7):819-23. doi: 10.1007/ s12603-018-1014-8

19. Toyoshima K, Nakamura M, Adachi Y, Adachi Y, Imaizumi A, Hakamada T, Abe Y, et al. Increased Plasma Proline Concentrations are Associated With Sarcopenia in the Elderly. PloS One (2017) 12(9):e0185206. doi: 10.1371/ journal.pone. 0185206

20. Lu Y, Karagounis LG, Ng TP, Carre C, Narang V, Wong G, et al. Systemic and Metabolic Signature of Sarcopenia in Community-Dwelling Older Adults. J Gerontol A Biol Sci Med Sci (2020) 75(2):309-17. doi: 10.1093/gerona/glz001

21. Ottestad I, Ulven SM, Øyri LKL, Sandvei KS, Gjevestad GO, Bye A, et al. Reduced Plasma Concentration of Branched-Chain Amino Acids in Sarcopenic Older Subjects: A Cross-Sectional Study. Br J Nutr (2018) 120(4):445-53. doi: 10.1017/S0007114518001307

22. Calvani R, Picca A, Marini F, Biancolillo A, Gervasoni J, Persichilli S, et al. A Distinct Pattern of Circulating Amino Acids Characterizes Older Persons With Physical Frailty and Sarcopenia: Results From the BIOSPHERE Study. Nutrients (2018) 10(11):1691. doi: 10.3390/nu10111691

23. Toshima T, Shirabe K, Kurihara T, Itoh S, Harimoto N, Ikegami T, et al. Profile of Plasma Amino Acids Values as a Predictor of Sepsis in Patients Following Living Donor Liver Transplantation: Special Reference to Sarcopenia and Postoperative Early Nutrition. Hepatol Res (2015) 45 (12):1170-7. doi: 10.1111/hepr.12484

24. Børsheim E, Bui QU, Tissier S, Kobayashi H, Ferrando AA, Wolfe RR. Effect of Amino Acid Supplementation on Muscle Mass, Strength and Physical Function in Elderly. Clin Nutr (2008) 27(2):189-95. doi: 10.1016/j.clnu.2008.01.001

25. Davis TA, Fiorotto ML, Burrin DG, Reeds PJ, Nguyen HV, Beckett PR, et al. Stimulation of Protein Synthesis by Both Insulin and Amino Acids Is Unique to Skeletal Muscle in Neonatal Pigs. Am J Physiol Endocrinol Metab (2002) 282 (4):E880-90. doi: 10.1152/ajpendo.00517.2001

26. CLaufenberg LJ, Pruznak AM, Navaratnarajah M, Lang CH. Sepsis-Induced Changes in Amino Acid Transporters and Leucine Signaling via mTOR in Skeletal Muscle. Amino Acids (2014) 46(12):2787-98. doi: 10.1007/s00726014-1836-6

27. Wu G. Amino Acids: Metabolism, Functions, and Nutrition. Amino Acids (2009) 37(1):1-17. doi: 10.1007/s00726-009-0269-0

28. Carlin MB, Tanner RE, Agergaard J, McClain DA, Drummond MJ. Skeletal Muscle Ras-Related GTP Binding B mRNA and Protein Expression is Increased After Essential Amino Acid Ingestion in Healthy Humans. J Nutr (2014) 144(9):1409-14. doi: 10.3945/jn.114.196691

29. Chantranupong L, Wolfson RL, Orozco JM, Saxton RA, Scaria SM, Bar-Peled L, et al. The Sestrins Interact With GATOR2 to Negatively Regulate the 
Amino-Acid-Sensing Pathway Upstream of Mtorcl. Cell Rep (2014) 9(1):1-8. doi: 10.1016/j.celrep.2014.09.014

30. Parmigiani A, Nourbakhsh A, Ding B, Wang W, Kim YC, Akopiants K, et al. Sestrins Inhibit Mtorc1 Kinase Activation Through the GATOR Complex. Cell Rep (2014) 9(4):1281-91. doi: 10.1016/j.celrep.2014.10.019

31. Wolfson RL, Chantranupong L, Saxton RA, Shen K, Scaria SM, Cantor JR, et al. Sestrin2 is a Leucine Sensor for the Mtorc1 Pathway. Science (2016) 351 (6268):43-8. doi: 10.1126/science.aab2674

32. Casperson SL, Sheffield-Moore M, Hewlings SJ, Paddon-Jones D. Leucine Supplementation Chronically Improves Muscle Protein Synthesis in Older Adults Consuming the RDA for Protein. Clin Nutr (2012) 31(4):512-9. doi: 10.1016/j.clnu.2012.01.005

33. Stipanuk MH. Leucine and Protein Synthesis: mTOR and Beyond. Nutr Rev (2007) 65(3):122-9. doi: 10.1111/j.1753-4887.2007.tb00289.x

34. Wang X, Proud CG. The mTOR Pathway in the Control of Protein Synthesis. Physiol (Bethesda) (2006) 21:362-9. doi: 10.1152/physiol.00024.2006

35. Liu S, Sun Y, Zhao R, Wang Y, Zhang W, Pang W. Isoleucine Increases Muscle Mass Through Promoting Myogenesis and Intramyocellular Fat Deposition. Food Funct (2021) 12(1):144-53. doi: 10.1039/D0FO02156C

36. Katsanos CS, Kobayashi H, Sheffield-Moore M, Aarsland A, Wolfe RR. A High Proportion of Leucine is Required for Optimal Stimulation of the Rate of Muscle Protein Synthesis by Essential Amino Acids in the Elderly. Am J Physiol Endocrinol Metab (2006) 291(2):E381-7. doi: 10.1152/ajpendo.00488.2005

37. Kim HK, Suzuki T, Saito K, Yoshida H, Kobayashi H, Kato H, et al. Effects of Exercise and Amino Acid Supplementation on Body Composition and Physical Function in Community-Dwelling Elderly Japanese Sarcopenic Women: A Randomized Controlled Trial. J Am Geriatr Soc (2012) 60(1):16-23. doi: 10.1111/j.1532-5415.2011.03776.x

38. Lin FD, Smith TK, Bayley HS. A Role for Tryptophan in Regulation of Protein Synthesis in Porcine Muscle. J Nutr (1988) 118(4):445-9. doi: 10.1093/jn/118.4.445

39. Dukes A, Davis C, El Refaey M, Upadhyay S, Mork S, Arounleut P, et al. The Aromatic Amino Acid Tryptophan Stimulates Skeletal Muscle IGF1/p70s6k/
mTor Signaling In Vivo and the Expression of Myogenic Genes In Vitro. Nutrition (2015) 31(7-8):1018-24. doi: 10.1016/j.nut.2015.02.011

40. Caballero B, Gleason RE, Wurtman RJ. Plasma Amino Acid Concentrations in Healthy Elderly Men and Women. Am J Clin Nutr (1991) 53(5):1249-52. doi: 10.1093/ajen/53.5.1249

41. Mayhew AJ, Amog K, Phillips S, Parise G, McNicholas PD, de Souza RJ, et al. The Prevalence of Sarcopenia in Community-Dwelling Older Adults, an Exploration of Differences Between Studies and Within Definitions: A Systematic Review and Meta-Analyses. Age Ageing (2019) 48(1):48-56. doi: 10.1093/ageing/afy106

42. Baracos VE. Psoas as a Sentinel Muscle for Sarcopenia: A Flawed Premise. J Cachexia Sarcopenia Muscle (2017) 8(4):527-8. doi: 10.1002/jcsm.12221

43. Brennan L. NMR-Based Metabolomics: From Sample Preparation to Applications in Nutrition Research. Prog Nucl Magn Reson Spectrosc (2014) 83:42-9. doi: 10.1016/j.pnmrs.2014.09.001

Conflict of Interest: The authors declare that the research was conducted in the absence of any commercial or financial relationships that could be construed as a potential conflict of interest.

Publisher's Note: All claims expressed in this article are solely those of the authors and do not necessarily represent those of their affiliated organizations, or those of the publisher, the editors and the reviewers. Any product that may be evaluated in this article, or claim that may be made by its manufacturer, is not guaranteed or endorsed by the publisher.

Copyright (c) 2021 Dai, Lin, Yue and Dai. This is an open-access article distributed under the terms of the Creative Commons Attribution License (CC BY). The use, distribution or reproduction in other forums is permitted, provided the original author(s) and the copyright owner(s) are credited and that the original publication in this journal is cited, in accordance with accepted academic practice. No use, distribution or reproduction is permitted which does not comply with these terms. 